\title{
THE METHODS OF CALCULATION \\ OF THE TIME OPTIMAL CONTROL OF LIGHT BRIDGE CRANES AT TRANSFERENCE OF LOAD ON FLEXIBLE ROPE SUSPENSION WITH VARYING LENGTHS
}

\author{
Streltsov P. M., Lehetska I. P., Labunska T. S.
}

\section{INTRODUCTION}

The volumes of the sea freight traffic in spite of crisis phenomena in the world economics continue their growth. First of all, it concerns cools for power engineering and big shipping containers. Between the main regions of economics development, the considerable cargo traffic is established. In such conditions when the full charging of ships of any freight capacity can be guaranteed, economics requirements force to put in exploitation the more and more big ships. Now gigantic ships are capable of carrying $200000 \mathrm{t}$ of bulk cargo and 16000 standard containers (TEU) become a dominant type of ships in the structure of the world merchant fleet.

In cargo handling of such ships, the berth reloaders with traditional construction scheme are used. The reloading comprises a high portal with fixed to it cantilever horizontal beam along which a trolley with cargo (a grab or a container) suspended to it with help of wire ropes travels. Geometrical parameters of the reloader are set in dependence of ship dimensions.

To minimize expenses connected with demurrage of ship user loading operation, the manufacturers, for instance, grab encoder the designers are trying to increase till $2000 \mathrm{t} / \mathrm{h}$ by increasing nominal velocities of the trolley of a modern reloader may be equal to $300 \mathrm{~m} / \mathrm{s}$ with acceleration equal to $1,4 \mathrm{~m} / \mathrm{s}^{2}$. With such parameters, the time of working cycle can be shortened to $45 \mathrm{~s}$.

The work of such reloader operators is very stressed. This includes making many mistakes such as not optimal combination of working movements, ineffective dampening of cargo oscillations etc. The quantity of such mistakes are increasing due to fatigue of crane driver and lead to decline of his average productivity almost by $20 \%$. The driver working on the limit of his possibilities become, as it is said, "physiological barrier" on the way to increasing the crane productivity. One of the means to surmount that barrier is automation of reloading machines which permits to essentially improve and "humanize" conditions of operator works and optimize working regime of machine and provide its work with constant high productivity independently from qualification of a driver, state of his health and other conditions. ${ }^{1}$

\footnotetext{
${ }^{1}$ Chernousjko F. L. Upravlenie. Moskva : Nauka, 1980. 384 p.
} 
In present time practically all new grab and many container reloaders are equipped with "electronic" systems of the automatic control. One of the tasks of such systems is dampening cargo oscillations.

The main element of such a system is an in-board computer. Dampening the trolley control is calculated previously and then allocated in memory of the computer, which provides realization of that law.

\section{SIMULATION MODEL OF LIGHT BRIDGE CRANE TRANSPORTED LOAD ON THE ROPE WITH VARYING LANE}

The aim of the proposed investigation is working out methods of calculation of time optimal or near to its laws of the control of the reloading cargo trolley, which transfers cargo suspended on the rope with the varying length at present distance (with simultaneous dampening of cargo oscillations).

The main method of investigation is mathematical simulation of the trolley movement. For definition of parameters of required controls the methods of the theory of the optimal control of movement of mechanical systems known as a "principle of maximum by Pontryagin" were used.

During such a research, the set of equations describing movement of the trolley with suspended cargo is used as a simulation model; the calculation scheme of the equivalent system under consideration is shown in Fig. 1.

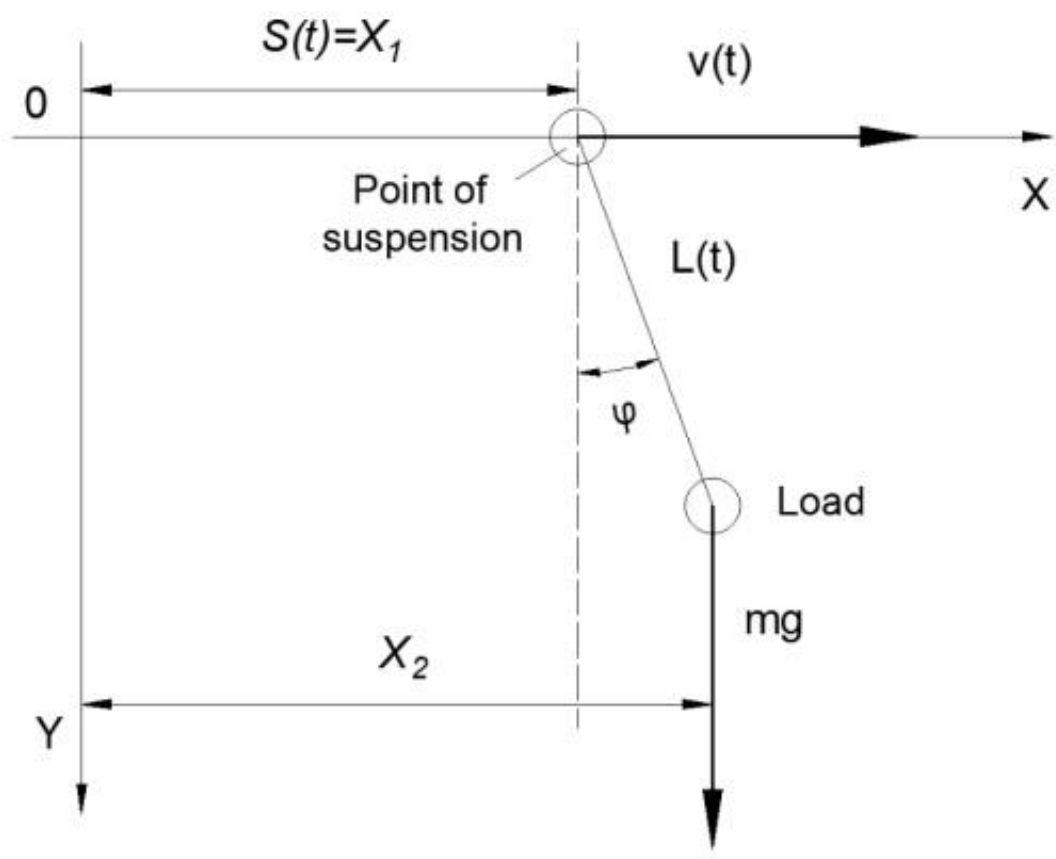

Fig. 1

The scheme comprises of point of suspension, which moves horizontally in accordance with definite law $s(t)$ and connected cargo with mass $m$ to it by weightless inextensible thread. In majority of cases work conditions of crane 
permit to set the length of thread as some time function 1(t). Oscillations of cargo are induced by stationary potential force (force of gravity).

The system has one degree of freedom with generalized coordinate $\phi$ (angle of rope declination from vertical).

For derivation of equation of movement of such system, the equation of Lagrange can be used:

$$
\frac{d}{d t} \cdot \frac{\partial L}{\partial y^{\prime}}-\frac{\partial L}{\partial \varphi}=0
$$

where L-function of Lagrange is equal to difference between kinetic $\mathrm{T}$ and potential $\Pi$ energy of the system.

Horizontal $X_{c}$ and vertical $X_{c}$ coordinates of cargo are defined by expressions:

$$
\begin{aligned}
& x_{c}=s(t)+l(t) \sin \varphi ; \\
& y_{c}=l(t) \cos \varphi .
\end{aligned}
$$

Equations for horizontal and vertical velocities of cargo are

$$
\begin{aligned}
& x_{c}=s^{\prime}+l^{\prime} \sin \varphi+l \varphi^{\prime} \cos \varphi, \\
& y_{c}^{\prime}=l^{\prime} \cos \varphi-l \varphi^{\prime} \sin \varphi .
\end{aligned}
$$

Potential energy of the system is:

$$
\Pi=-m_{g} y_{c}=-m g l \cos \varphi
$$

For kinetic energy after simple conversions:

$$
T=\frac{m}{2}\left(x_{c}^{\prime 2}+y_{c}^{\prime 2}\right)=\frac{m}{2}\left(s^{\prime 2}+l^{\prime 2}+l^{2} \varphi^{\prime 2}+2 l^{\prime} s^{\prime} \sin \varphi+2^{\prime} s^{\prime} \varphi^{\prime} \cos \varphi\right)
$$

The function of Lagrange

$$
L=T-\Pi=\frac{m}{2}\left(s^{\prime 2}+l^{\prime 2}+l^{2} \varphi^{\prime 2}+2 l^{\prime} s^{\prime} \sin \varphi+2 l s^{\prime} \varphi^{\prime} \cos \varphi\right)+m g l \cos \varphi
$$

Partial derivative of L by generalized velocity y':

$$
\frac{\partial L}{\partial \varphi^{\prime}}=m l^{2} \varphi^{\prime \prime}+m l s^{\prime} \cos \varphi
$$


Its derivative in time

$$
\frac{d}{d t} \cdot \frac{\partial L}{\partial \varphi^{\prime}}=2 m l l^{\prime} \varphi^{\prime}+m l^{2} \varphi^{\prime \prime}+m l^{\prime} s^{\prime} \cos \varphi+m l s^{\prime \prime} \cos \varphi-m l s^{\prime} \varphi^{\prime} \sin \varphi
$$

Partial derivative of Lagrange function by generalized coordinate $\varphi$ :

$$
\frac{\partial L}{\partial \varphi}=m l^{\prime} s^{\prime} \cos \varphi-m l s^{\prime} \sin \varphi-m g l \sin \varphi
$$

After putting found expressions in equation (1) of Lagrange, and cutting of similar ones, we can receive:

$$
2 m l l^{\prime} \varphi^{\prime}+m l^{2} \varphi^{\prime \prime}+m l s^{\prime \prime} \cos \varphi+m g l \sin \varphi=0
$$

From that expression

$$
\varphi^{\prime \prime}=-\frac{s^{\prime \prime}}{l} \cos \varphi-\frac{g}{l} \sin \varphi-2 \frac{l^{\prime} \varphi^{\prime \prime}}{l}
$$

In received equation, which describes the movement of the point of suspension and cargo, the generalized coordinate $\varphi$ is used. But very often at investigation of cranes movement utilization of generalized coordinate $X$ instead of $\varphi$ is more convenient. Let us derive $X$ for definition of the state of the system (2)

Coordinates $\mathrm{X}$ and $\varphi$ are connected by the expression

$$
\sin \varphi=\frac{x}{l}
$$

Let us differentiate by time twice expression (12):

$$
\begin{aligned}
\varphi^{\prime} \cos \varphi & =\frac{x^{\prime}}{l}-\frac{x l^{\prime}}{l^{2}} ; \\
\varphi^{\prime \prime} \cos \varphi-\varphi^{\prime 2} \sin \varphi & =\frac{x^{\prime \prime}}{l}-2 \frac{x^{\prime} l^{\prime}}{l^{2}}-\frac{x l^{\prime \prime}}{l^{2}}+2 \frac{x l^{\prime 2}}{l^{3}}
\end{aligned}
$$

It is possible with help of received equation (13) to express derivative $\varphi^{\prime}$ and $\varphi^{\prime \prime}$ through new generalized coordinate $X$ and its derivative. After substitution in equation (11), the equation of Lagrange can be received in the next form: 


$$
\begin{aligned}
& \frac{x^{\prime}}{l \cos \varphi}-2 \frac{x^{\prime} l^{\prime}}{l^{2} \cos \varphi}-\frac{x l^{\prime}}{l^{2} \cos \varphi}+2 \frac{x l^{\prime 2}}{l^{3} \cos \varphi}+ \\
& +\frac{x^{2} \sin \varphi}{l^{2} \cos ^{2} \varphi}+\frac{x^{2} l^{\prime 2} \sin \varphi}{l^{\prime \prime} \cos \varphi}-2 \frac{x x^{\prime} l^{\prime} \sin \varphi}{l^{3} \cos ^{3} \varphi}= \\
& =-\frac{s^{\prime \prime}}{l} \cos \varphi-\frac{g}{l} \sin \varphi-2 \frac{x^{\prime} l^{\prime}}{l^{2} \cos \varphi}+2 \frac{x l^{\prime 2}}{l^{3} \cos \varphi}
\end{aligned}
$$

Omitting infinitesimal elements and taking into account that, due to the small angles of rope slopes, $\cos \varphi=1$ can be considered, the acceleration $\mathrm{x}^{\prime \prime}$ can be expressed in the following form:

$$
x^{\prime \prime}=-s^{\prime \prime}-\frac{g-l^{\prime \prime}+\frac{x^{\prime 2}}{l}}{l} x
$$

Thus, acceleration $\mathrm{x}^{\prime \prime}$ of cargo in relation to point of suspension is defined by acceleration $\mathrm{s}^{\prime \prime}$ of the point of suspension and absolute horizontal acceleration of cargo evoked by a horizontal component of the force in the rope. That force as can be seen from equation (15) in its turn depends on cargo weight, force of inertia caused by variation of the rope length with acceleration 1 " and centrifugal force called forth by acceleration $\frac{x^{12}}{l}$.

The obtained equation (15) can be used as a simulation mathematical model of the crane for determining rational (optimal or quasi optimal) controls of crane mechanisms.

Search of wanted controls for big cranes are connected with serious difficulties. At first, it is expedite to elaborate methods of determination of control for small hoisting machines such as light overhead (bridge) cranes which are often included in automatic systems performing various technological processes.

The control of such cranes is fulfilled with help of inboard or external computers.

Parameters of required controls are previously calculated in dependence of geometrical conditions of a duty cycle and thence are placed in the computer memory, which monitors realization of the calculated control in the automatic regime.

Light bridge cranes can be regarded as physical models of more powerful machines such as widely used in ports ship-to-shore quay and yard grab or container cranes. 
Methods of calculation and automatic realization of controls thoroughly tested on small cranes can be used for automation and optimization of work of large machines and permit to increase their productivity and provide noticeable economical effect.

Setting the task

Oscillations of load on the rope, caused by the trolley movement, create additional difficulties in realization of the control and increase time of crane duty cycle.

The calculated control must provide dampening such oscillations at approach of the trolley to the target point of its way.

Methods of calculations of time optimal or near to it by effective kinds of the control for transportation of load on the rope with the fixed length are worked out deep by enough ${ }^{2}$.

In real conditions for decreasing time of a duty cycle, crane operators combine horizontal movement of load with its hoisting (lowering). But questions of optimization of the crane control for transportation of load hanging on the rope with the varying length are investigated insufficiently (some aspects of that problem are regarded in works ${ }^{3},{ }^{4}$.

The proposed materials are dedicated to elaboration of methods of calculation of time optimal or near to it by effective kinds of the control for light bridge cranes during transportation of load hanging on the rope with the varying length.

Content and results of the investigation

The calculation scheme for such a crane can be represented by mechanical system shown in fig. 1 .

The characteristic feature of the motor practically immediately begins to move with a set velocity and at switching off stops instantly without further motion. That feature permits to utilize velocity $V(t)$ of the trolley as its control parameter.

To avoid excessive dynamic stresses and increase fatigue life it is desirable not to change a sign of velocity (i.e. permit movement only in one direction to the target point). Taking it into account, restriction on the control parameter can be written in the next form:

$$
0 \leq \mathrm{V}(\mathrm{t}) \leq \mathrm{V}_{\max }
$$

2 Strel'tsov P. M. Gashenie kolebaniy gruza pri ego peremeshchenii na podvese peremennoy dliny. Visnyk ONMU. 2012. № 35. Pp. 179-189.

${ }^{3}$ Verschov T. Cranes-Design,Practise and Maintenance (Chapter 6. Sway and Swiny; Automation the trolley travelling mechanism). Professional Engineering Publishing. 2002. Pp. 167-173.

${ }^{4}$ Boltyanskiy V. G. Matematicheskie metody optimal'nogo upravleniya. Moskva : Nauka, 1969. Pp. 408. 
The movement of load is defined by the pull force in the inclined rope. As it can be seen from equation (15) the movement of regarded material system "trolley-load" can be written in the next form:

$$
\begin{aligned}
& X_{T}^{\prime}=V ; \\
& X_{C}^{\prime \prime}=g\left(X_{T}-X_{C}\right) / l(t),
\end{aligned}
$$

where $X_{T}$-coordinate of the trolley; $X_{c}$ - coordinate of cargo.

Introduction of new variables $X_{1}=X_{T} ; X_{2}=X_{C} ; X_{3}=X_{C}$ permits to write equations (17) in the normal form as a system of three differential equations of the first degree:

$$
\begin{aligned}
& X_{1}^{\prime}=V ; \\
& X_{2}^{\prime}=X_{3} ; \\
& X_{3}^{\prime}=g\left(X_{1}-X_{2}\right) / l(t) .
\end{aligned}
$$

Represented equations can be regarded as a mathematical (simulation) model of the investigated mechanical system. That model can be utilized for determination of laws of changing the velocity $\mathrm{V}(\mathrm{t})$ of the trolley, which provides desirable character of movement of the system "trolley-load".

Let us suppose that movement of the trolley begins from the state of rest, defined by the next coordinates.

$$
X_{1}^{B}=0 ; X_{2}^{B}=0 ; X_{3}^{B}=0 .
$$

The trolley will come after transference at distance $S$ to the target position of rest with next coordinates:

$$
X_{1}^{E}=0 ; X_{2}^{E}=0 ; X_{3}^{E}=0 .
$$

The problem for search of optimal $\mathrm{V}^{*}(\mathrm{t})$ for such transition can be set in the next form.

It is necessary to determine control $\mathrm{V}^{*}(\mathrm{t})$ which provides transition of a mechanical system described by equations (18) from the beginning state (19) to the set end state (20) by the shortest time under observance of restrictions (16) for control parameter $\mathrm{V}(\mathrm{t})$.

To ascertain the character of the time optimal control, the mathematical theory of the optimal control known as a "principle of maximum by Pontryagin" may be used ${ }^{5}$. In accordance with that theory the function $\mathrm{H}$ of

${ }^{5}$ Vasil'ev F. P. Chislennye metody resheniya ekstremal'nykh zadach. Moskva : Nauka, 1988-545 pp. 
Hamilton for the investigated mechanical system based on equations of its mathematical model must be written. For our case, the function of Hamilton is:

$$
H=\sum \varphi_{i} X_{i}^{\prime}=\varphi_{1} V+\varphi_{2} X_{3}+\varphi_{3} g\left(X_{1}-X_{2}\right) / l(t)
$$

The auxiliary functions $\varphi_{\mathrm{i}}$, which Hamiltonian $\mathrm{H}$ includes, are defined by the formula

$$
\varphi_{i}^{\prime}=-\frac{\partial H}{\partial X_{i}}
$$

Therefore we have

$$
\varphi_{i}^{\prime}=-\varphi_{3} g / l(t) ; \varphi_{2}^{\prime}=\varphi_{3} g / l(t) ; \varphi_{3}^{\prime}=-\varphi_{2} .
$$

From system (23) for auxiliary function $\varphi$, the formula can be received

$$
\varphi_{i}^{(3)}+2 \varphi_{1}^{\prime \prime} l^{\prime} / l(t)+\varphi_{1}^{\prime} g / l(t)=0 .
$$

In accordance with the "principle of maximum" the optimal control must provide the maximum to Hamiltonian $\mathrm{H}$. As it can be seen from expression (21), Hamiltonian will achieve maximum, if at positive meanings of function $\varphi_{1}$ the control function will take its maximal value (i.e. $V=\mathrm{V}_{\max }$ ) and at negative $\varphi_{1}$ control function $V$ will take its minimal value $V=0$.

Thus, optimal control $\mathrm{V}^{*}(\mathrm{t})$ is a relay (piecemeal-constant) function and takes on intervals of constancy extreme values. The moments of switching off the control from one extreme value to another correspond to the points of crossing the axis of abscissae by the graph of auxiliary function $\varphi_{1}$.

The quantity and duration of intervals of the optimal control are defined by location of the graph of function $\varphi_{1}$ relatively to the axis of abscissae.

Let us consider the methods of determination of the optimal control of the trolley at transportation of cargo on the rope with the constant length. In that case equation (23) takes form $\boldsymbol{\varphi}_{\mathbf{1}}{ }^{(3)}+\mathbf{k}^{2} \boldsymbol{\varphi}_{\mathbf{1}}{ }^{\prime}=\mathbf{0},(24)$ where $\mathrm{k}^{2}=\mathrm{g} / \mathrm{l}$

Solution of that uniform linear differential equation is the following:

$$
\begin{aligned}
& \varphi_{1}=A \sin (k t+\alpha)+B ; \\
& \varphi_{1}^{\prime}=A k \cos (k t+\alpha) ; \\
& \varphi_{1}^{\prime \prime}=-A k^{2} \sin (k t+\alpha) .
\end{aligned}
$$

where $\alpha$ - beginning phase of function $\varphi_{1} ; \mathrm{A}-$ amplitude of function $\varphi_{1}$; $\mathrm{B}$ - vertical displacement of the axis of sinusoid is relatively to the axis of abscissae. 


\section{NUMERICAL METHODS OF CALCULATION OF THE TIME OPTIMAL CONTROL OF LIGHT BRIDGE CRANE}

Possible graph of auxiliary function $\varphi_{1}$ is shown in fig. 2. As it was found, optimal control $\mathrm{V} *(\mathrm{t})$ of the investigated material system is a piecemeal constant function, which includes some quantity of intervals of constancy. At each interval function $\mathrm{V}^{*}(\mathrm{t})$ preserves the boundary value (maximal $\mathrm{V}(\mathrm{t})=\mathrm{V}_{\max }$ or minimal $\left.\mathrm{V}(\mathrm{t})=0\right)$.

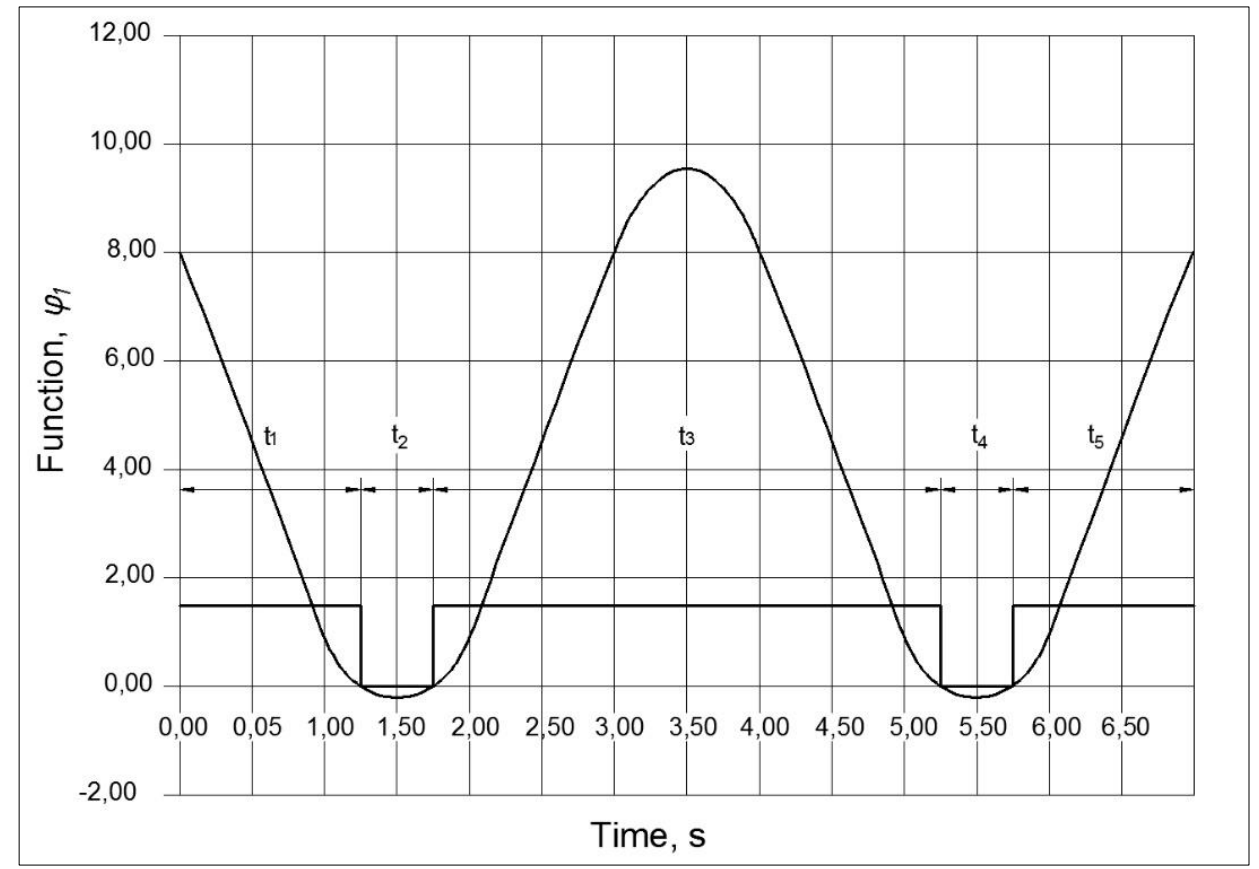

Fig. 2

The "principle of maximum" permits to define only the general notion about the optimal control. But the quantity of intervals and their durations have to be defined by special procedure.

With that aim let us consider the character of cargo oscillation on the rope with the constant length at optimal control $V^{*}(t)$.

As acceleration of point of suspension is equal to zero $X_{1}^{\prime \prime}=0$, the third equation of mathematical model (17) can be rewritten in the next form:

$$
X_{1}^{\prime \prime}-X_{2}^{\prime \prime}=-\left(X_{1}-X_{2}\right) g / l
$$

with new designations, $y=X_{1}-X_{2}, k^{2}=g / l$ equation (26) will be the following:

$$
y^{\prime \prime}+k^{2} y=0
$$


The solution of that uniform linear differential equation is the following:

$$
\begin{gathered}
y=A \cos k t+B \sin k t \\
y^{\prime}=-A k \sin k t+B k \cos k t .
\end{gathered}
$$

As the movement of the system starts from the state of rest when load declination from vertical is y $(0)=0$ and the velocity of declination is $\mathrm{y}^{\prime}(0)=-$ $\mathrm{V}_{\max }$ for constants $\mathrm{A}$ and $\mathrm{B}$ of integration we shall receive

$\mathrm{A}=0 ; \mathrm{B}=-\mathrm{V}_{\max } / \mathrm{k}$.

The movement of load relatively to the point of suspension will be described by equations:

$$
\begin{aligned}
& y^{(t)}=-\left(V_{\max } / k\right) \sin k t \\
& y^{\prime}(t)=-V_{\max }
\end{aligned}
$$

i.e. at the movement of the point of suspension with constant velocity $\mathrm{V}_{\max }$ the load will fulfil harmonic oscillation relatively to the vertical line which crosses the point of suspension (state of equilibrium). The period of oscillations is:

$$
\tau=2 \pi / k=2 \sqrt{l}
$$

amplitude of declination $y=V_{\max } / k$

Function $\varphi_{1}$ (fig.2) is a sinusoid with the period equal to the period of load oscillations $\tau=2 \pi / k=2 \sqrt{l}$

Each time when the graph of sinusoid crosses the axis of time, the switching off control function $\mathrm{V}^{*}(\mathrm{t})$ is fulfilled.

So, the quantity of switching off the optimal control depends on duration $\mathrm{T}$ of displacement of the system. If $\mathrm{T} \leq \varepsilon$, the graph of $\varphi_{1}$ will be a partial wave of the sinusoid which crosses the axis of time only in two points. And optimal control $\mathrm{V}^{*}(\mathrm{t})$ will have only three intervals. If $\tau \leq \mathrm{T} \leq 2 \tau$ graph of $\varphi_{1}$ will include the whole wave of the sinusoid and cross axis of time in four points, so the control will consist of five intervals. At $2 \tau \leq \mathrm{T} \leq 3 \tau$, the control will have seven intervals and so on.

Below the method of definition of time parameters (quality and duration of intervals) of the control is considered for the next geometrical conditions:

- the length of rope $t=4 t$;

- the period of oscillation $\tau=4 \mathrm{~s}$;

- the maximal velocity of the trolley $\mathrm{V}_{\max }=0,3 \mathrm{t} / \mathrm{s}$;

- the distance of load displacement $\mathrm{S}=1,8 \mathrm{~m}$. 
In that case the total duration of intervals, at which the movement of the trolley is fulfilled, is equal $\mathrm{S} / \mathrm{V}=1,8 / 0,3=6 \mathrm{~s}$.

Hence, the time of displacement $\mathrm{T} \tau \leq \mathrm{T} \leq 2 \tau$, in that case, as was proved above, the graph of function $\varphi_{1}$ will cross axis of time in four points and quality of intervals will be equal to five.

As the beginning and end conditions of movement are symmetrical, graphs of optimal control $\mathrm{V}^{*}(\mathrm{t})$ and function $\varphi_{1}$ will be symmetrical in relation to their middle (as shown at fig.2)

If to mark durations of the first and second intervals as $t_{1}$ and $t_{2}$, the duration of the third (middle) will be $\mathrm{t}_{3}=\tau-\mathrm{t}_{2}$; fourth and fifth (last) will be $\mathrm{t}_{4}=\mathrm{t}_{2}$, $\mathrm{t}_{5}=\mathrm{t}_{1}$.

As the displacement of the trolley is fulfilled only at odd intervals, their total duration must be equal to $6 \mathrm{~s}$ :

$$
t_{1}+t_{2}+t_{3}=t_{1}+\tau-t_{2}+t_{1}=S / V_{\max }=6 s,
$$

hence

$$
t_{2}=2 t_{1}-2
$$

For material systems similar to investigated ones, the movement of which is described by linear differential equation, the principle of superposition can be used. In accordance with its oscillation, the load is a sum of oscillations, which arise at the moments of switching off the control parameter.

In the end point, the moving system has to be in a state of rest, i.e. deflection of load and velocity y' of its changing must be equal to zero.

It is possible in accordance with a principle of superposition for load deflection at moment $\mathrm{T}$ of movement ending for deflection y to write (taking into account expression (32)):

$$
\begin{aligned}
& y(T) \cdot k / V_{\max }=-\sin k\left(2 t_{1}+\varepsilon+t_{2}\right)+\sin k\left(t_{1}+t_{2}+\varepsilon\right)- \\
& -\sin k\left(\tau+t_{1}\right)+\sin k\left(t_{1}+t_{2}\right)-\sin k t_{1}+\sin k \times 0= \\
& =-\sin k\left(4 t_{1}-2\right)+\sin k\left(3 t_{1}-2\right)-\sin k t_{1}+\sin \left(3 t_{1}-2\right)- \\
& -\sin k t_{1}+\sin k \times 0=-\sin k\left(4 t_{1}-2\right)+2 \sin k\left(3 t_{1}-2\right)- \\
& -2 \sin k t_{1}+\sin k \times 0=0
\end{aligned}
$$

For velocity $y^{\prime}$ of changing the deflection at moment $T$ after differentiation, it is possible to receive:

$$
\begin{aligned}
& y^{\prime}(T) / V_{\max }=-\cos k\left(4 t_{1}-2\right)+2 \cos k\left(3 t_{1}-2\right)- \\
& -2 \cos k t_{1}+\cos k \times 0=0
\end{aligned}
$$


For the solution of received trigonometric equations it is possible to use a simple enough numerical method. Algorithm of method requires changing from " 0 " of value of $t_{1}$ with definite rational step. At each step meaning of sums (33) and (34) are calculated. As solution must be taken, value of $t_{1}$, at which both sums simultaneously come near enough to " 0 ". The performed calculation shows that such condition is fulfilled at $\mathrm{t}_{1}=1,25 \mathrm{~s}$.

Durations of following intervals are:

- second interval $\mathrm{t}_{2}=0,5 \mathrm{~s}$;

- third (middle) $t_{3}=3,5 \mathrm{~s}$;

- fourth $\mathrm{t}_{4}=0,5 \mathrm{~s}$;

- fifth (east) $\mathrm{t}_{5}=1,25 \mathrm{~s}$.

By results of calculations graphs of auxiliary function $\varphi_{1}$ and optimal control $\mathrm{V}^{*}(\mathrm{t})$ has been built (see fig. 2).

For definition of position of function $\varphi_{1}$ relatively to time axis, another method, which has more general character, can be used.

That method can be used for calculations of the optimal control in cases when function $\varphi_{1}$ is described by nonlinear differential equations (for instance in cases of crane movement with the varying rope length).

In that case, time parameters of the optimal control are defined by equation (24).

After introduction of new variables

$$
f_{1}=\varphi_{1} ; f_{2}=\varphi_{1}^{\prime} ; f_{3}=\varphi_{1}^{\prime \prime}
$$

equation (9) can be re-written in a normal form as a system of three differential equations of the first degree

$$
\begin{aligned}
& f_{1}^{\prime}=f_{2} \\
& f_{2}^{\prime}=f_{3} \\
& f_{3}^{\prime}=-\left(2 l^{\prime} f_{3}+g f_{2}\right) / l(t) .
\end{aligned}
$$

It is necessary for digital integration of system (36) to present initial values of functions $\mathrm{f}_{1}, \mathrm{f}_{2}, \mathrm{f}_{3}$. For that aim let us investigate small enough initial section of the trajectory of the mechanical system "trolley-load" (18) for which the length of the rope can be regarded as fixed.

Equation (36) for that section can be written in the following form

$$
\varphi_{1}^{(3)}+\varphi_{1}^{\prime} g / l^{B}=0
$$

where $l^{B}$ - initial value of the rope length. 
The solution of that linear differential equation has the following form:

$$
\varphi_{1}=A \sin \left(\sqrt{\frac{g}{l^{B}}} \bullet t+\alpha\right)+B
$$

where $\varphi$-initial phase of function $\varphi_{1}$;

B-distance between the axis of function $\varphi 1$ and the axis of abscissae A-amplitude of function $\varphi_{1}$.

As it can be shown from equation (10) initial values of functions $f_{1}^{\prime \prime}, f_{2}^{\prime \prime}, f_{3}^{\prime \prime}$ are connected with parameters $\alpha, \mathrm{A}$ and $\mathrm{B}$ by expressions

$$
f_{1}^{\prime \prime}=A \sin \alpha+B ; f_{2}^{\prime \prime}=\sqrt{\frac{g}{l^{H}}} ; f_{3}^{\prime \prime}=-\frac{g}{l^{H}} \sin \alpha
$$

Thus, location of the graph of function $\varphi_{1}$ (hence, character of the optimal control) is defined by choice of parameter $\alpha, \mathrm{A}$ and $\mathrm{B}$.

It is expedient to choose as a criterion of optimality for such choice the value of energy $E$ of residual sway defined by the formula (aim function):

$$
E=\left(X_{3}^{E}\right)^{2}+g\left(X_{1}^{E}-X_{2}^{E}\right)^{2} / L^{E}
$$

Here the first item is proportional to the kinetic energy and the second is proportional to the potential energy of swaying load.

The calculations of values of aim function $\mathrm{E}$ are connected with complicated enough procedure, which includes numerical integration of equations of mathematical model (3) under the set control from the initial one to the target point of the trolley way.

In case, when the set control is optimal, the energy of residual sway will be equal to zero.

Thus, the task about the search of the function of control $\mathrm{V}^{*} / \mathrm{t}$ which provides the minimal time of the load transportation is reduced to the task about determination of such values of only three parameters $(\alpha, A$ and $B)$ which provide minimization of aim function $\mathrm{E}$.

To solve that task a special algorithm is proposed. According to the algorithm, it is necessary to fulfil the next steps:

1) to set values of parameters $\alpha, A$ and $B$;

2) to determine initial values of function $\varphi_{1}$ and its derivatives;

3) to carry out digital integration of equation (36), and then determine function $\varphi_{1}$ and corresponding to its control $\mathrm{V}(\mathrm{t})$;

4) to fulfill the integration of equation (18) of the mathematical model of the trolley under determined control V ( $t$ ) and calculate the value of aim function $\mathrm{E}$ at the end of the trolley way; 
5) if $\mathrm{E}$ is too big, to change values of parameters $\alpha, \mathrm{A}$ and $\mathrm{B}$;

6) to fulfill described above the procedure with new values $\alpha$, A and B.

The iteration process described above must be continued till determination of such control $\mathrm{V} *(\mathrm{t})$ at which aim function $\mathrm{E}$ will become equal (or near enough) to zero.

On the base of that algorithm the program for numerical calculation of time optimal control $\mathrm{V}^{*}(\mathrm{t})$, which provides damping of load sway at the end of set distance, was worked out.

To confirm the effectiveness of the method and successful work of program graphs of movement parameters of the system "trolley-load" built by results of computer calculations of the optimal control are presented in fig. 2.

General sequence of the proposed solution of the considered problem corresponds to the numerical method of optimal problems solution known as the method of coordinate-by-coordinate descend. In accordance to that method, described for instance in ${ }^{6}$, the program of definition of optimal control $V^{*}(t)$ of the investigated system was worked out with help of program calculations of auxiliary function $\varphi_{1}$ of the optimal control and parameters of the movement of the material system (17) fulfilled for the same geometrical conditions as in the previous case.

It was turned out that the iteration process of searching aim function $\mathrm{E}$ extremum is converged soon enough.

It was worth noting that the proposed method (which has broader sphere of utilization) is difficult enough; requires utilization of complicated programs and big volumes of calculation. All that can evoke doubts in received results. To prove the reality of results the new method must be carefully tested for instance by comparing its results with solutions received by other methods thoroughly tested and reliable.

Graphs of movement parameters of designations correspond: 1- optimal control $\mathrm{V}^{*}(\mathrm{t})$ of the trolley (its velocity); 2 - absolute velocity of load; 3 deflexion of load from the state of equilibrium; 4 - length of rope; 5 - trolley transference; 6 - load transference; 7 - auxiliary function $\varphi_{1}$ of Hamiltonian. For convenience, the graphs of trolley and load velocities and deflexion (18) of load are presented on a large scale.

As can be seen in fig. 2 and 3 results of calculations of durations of individual intervals of the optimal control received by both methods (previous and described now) coincide.

\footnotetext{
${ }^{1}$ Nebesnov V. I. (1965) Voprosy sovmestnoy raboty dvigateley vintov i korpusa sudna [Issues of joint work of propeller engines and hull]. L.: Shipbuilding, 1965. 247 p.
} 


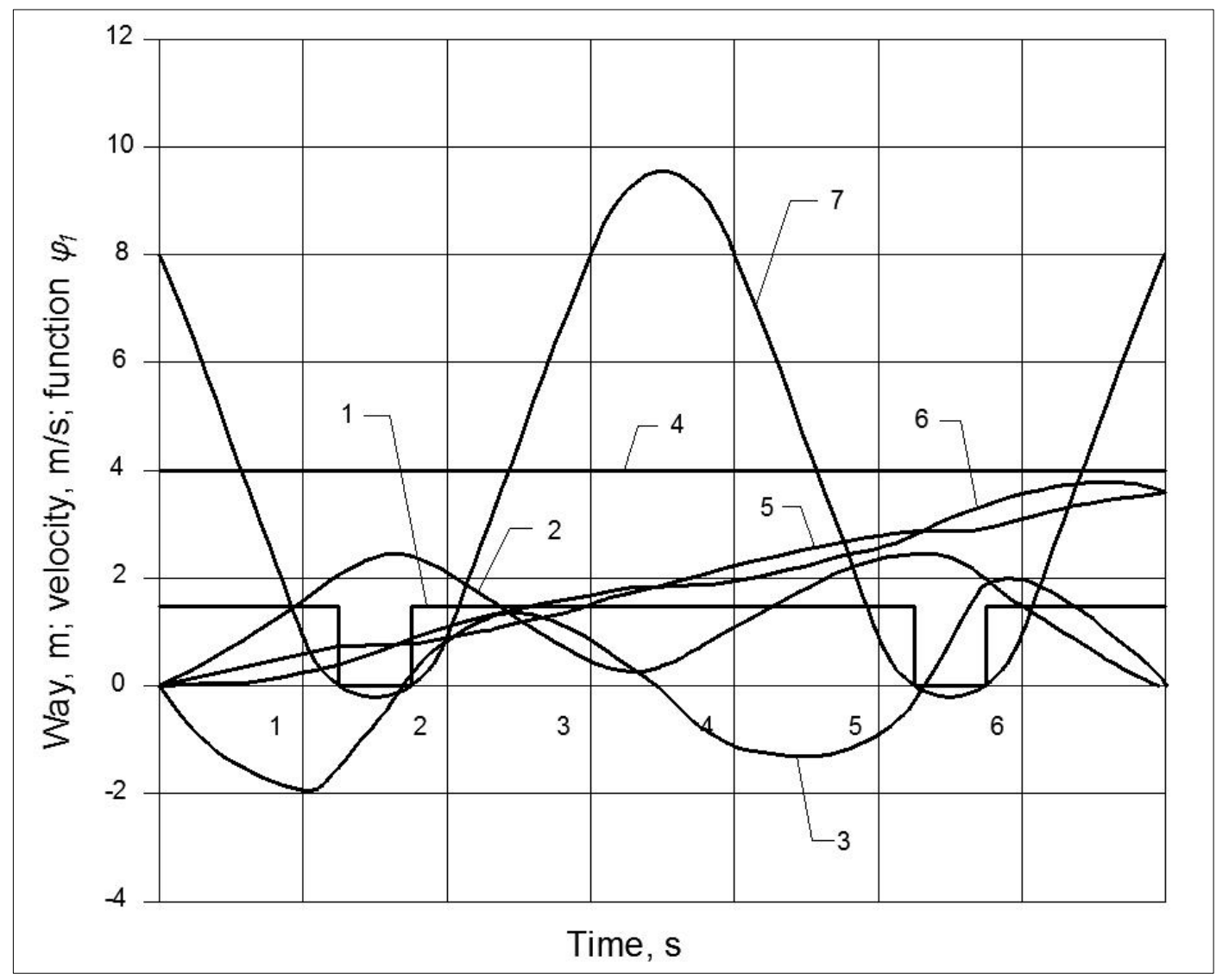

Fig. 3

Hence, the new method, which does not require linearity of mathematical model equations, permits to receive reliable enough results and can be used in more complicated cases. The calculations were fulfilled for the next conditions:

- the distance of the trolley and load movement $\mathrm{S}$

- the maximal velocity of the trolley $\mathrm{V}_{\max }=0,3 \mathrm{~m} / \mathrm{s}$

- the rate of the rope varying (hoist) $1(\mathrm{t})^{\prime}$

Let us consider the utilization of the new method for definition of the optimal control of the trolley at transference of load on the rope with the varying length (when equations of function $\varphi_{1}$ are non-linear, and the first of considered methods is not suitable), at the next geometric parameters of the movement:

- the set distance of the trolley $\mathrm{S}=1,8 \mathrm{~m}$;

- the limitation on control parameter V (the velocity of the trolley)

$$
0 \leq \mathrm{V}(\mathrm{t}) \leq \mathrm{V}_{\max }
$$

- the initial rope length $1(0)=4 \mathrm{~m}$;

- the length of the rope is changing from the initial value with constant velocity and can be presented as a function of time $1(t)=4-0,2 t$. 
In our case at defining the situation of the graph of function $\varphi_{1}$ a new member, which reflects the character of the rope length changing, are to be included in equations (23). That equation after inclusion of new variables in normal form (35) will have the next form

$$
\begin{aligned}
& f_{1}^{\prime}=f_{2} \\
& f_{2}^{\prime}=f_{3} \\
& f_{3}^{\prime}=-2 f_{3} l^{\prime}(t) / l(t)-f_{2} g / l(t)
\end{aligned}
$$

Initial values of functions $f_{1}(0) ; f_{2}(0) ; f_{3}(0)$ can be calculated with help of equations (37), where $k^{2}=g / l(0)$.

The same algorithm and the same program corrected with taking in account the character of the rope length changing were utilized for definition of the optimal control at shown above geometrical conditions.

The graphs of parameters of movement at the optimal control and the varying rope length built on the base of calculations with help of worked out program are presented in fig. 4 .

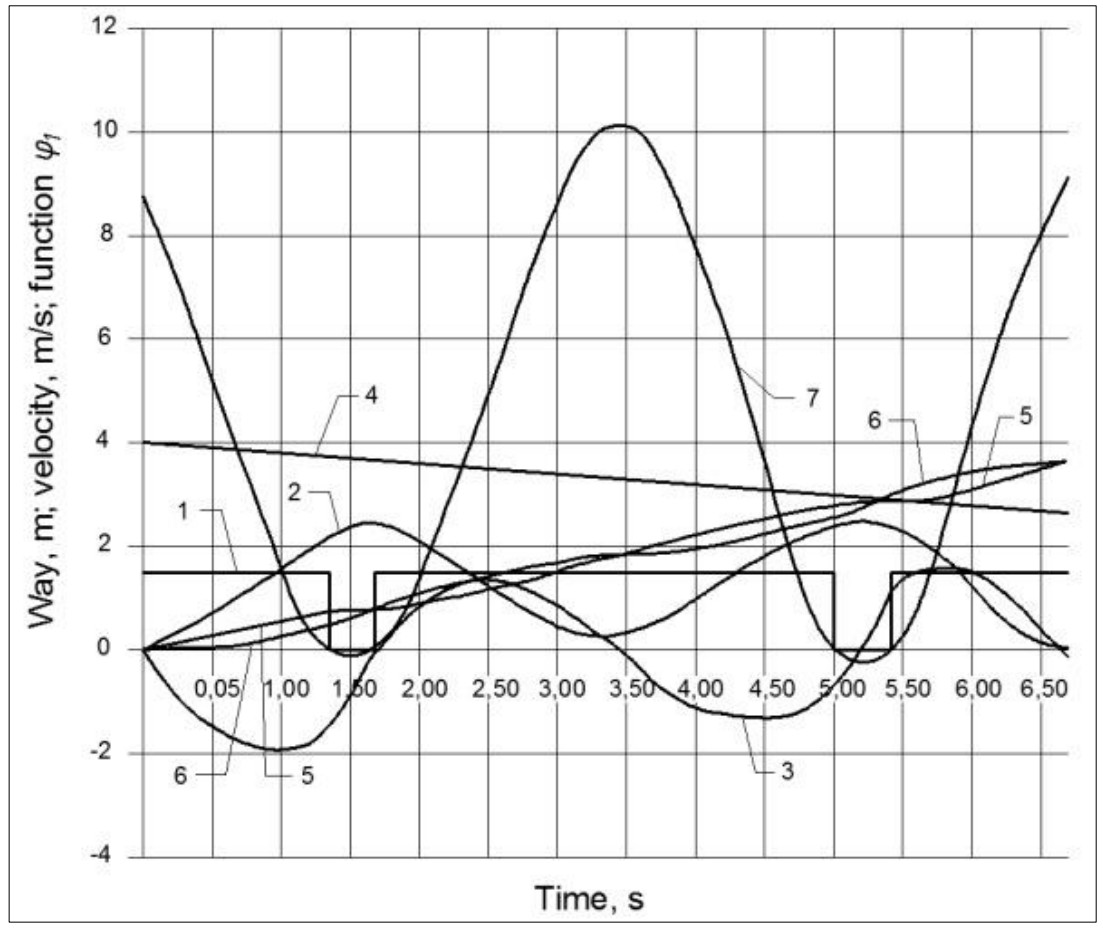

Fig. 4

The graphs confirm effectiveness of considered method and worked out the program, which permits to find controls of the material system (a trolley with load on the rope with the varying length), which corresponds to the necessary "time optimality condition" required by the "principle of maximum". 
As can be seen from the figure, auxiliary function $\varphi_{1}$ has oscillative character and can cross axis of time many times. The quantity of crossing points and hence quantities of intervals and points of switching off the control is increasing with increase of duration of transference and can be considerable enough, that can create difficulties at realization. In our case, the quantity of intervals in accordance with calculation occurred also to be equal to the fifth. Unlike the previous case, the even intervals do not have equal durations. The general duration of movement is equal to $6,66 \mathrm{~s}$.

The transference of the trolley is accompanied with intensive swaying of load with maximal amplitude equal to $0,2 \mathrm{~m}$.

Another kind of the control being more simple and convenient for realization can be proposed.

To avoid sways at the main part of the trolley way its movement can be organized in three stages.

At the first stage acceleration of load to horizontal velocity $\mathrm{V}_{\max }$ must be fulfilled in such a way that rope would have a vertical position at the end of acceleration.

At the second stage, the movement of the trolley must be continued with velocity $\mathrm{V}_{\max }$, which will move synchronously with the trolley. At that period, the changes of the rope length do not exert any influence on horizontal movement of load.

At the third stage, the horizontal velocity of load must be reduced to zero in such a way when the rope would be hanging vertically under the motionless trolley.

It is desirable that all stages have minimal durations. To achieve it, the character of the control on the stages of the transient movement must correspond to the "principle of maximum", i.e. to be defined by forms of Hamiltonian $H$ (1) and auxiliary function $\varphi_{1}$ (9). Hence, the time optimal control of the trolley at these stages must be also relay-functions which are taken at intervals of constancy values $\mathrm{V}=\mathrm{V}_{\max }$, when $\varphi_{1}>0$, and $\mathrm{V}=0$ when $\varphi_{1}<0$.

It is possible to prove that durations of stages of transient movement do not exceed the time of the half-cycle of load oscillation.

During that period function $\varphi_{1}$ can cross the axis of abscissae only once. The optimal control at such stages will have only two intervals (with total quantity of intervals equal to five).

To define durations of these intervals the same program, which was used for calculation of the optimal control with five intervals can be used again after some insignificant additions. With help of such corrected program the new kind of control $\mathrm{V}_{5}(\mathrm{t})$ which provides damping of load sway on transient stages and contain five intervals, was calculated for the same geometrical conditions as in previous cases. 
By results of calculation with help of program the graphs of parameters of the movement "trolley-load" system were built (see fig. 5). The designations in fig. 5 are the same as in fig. 3 .

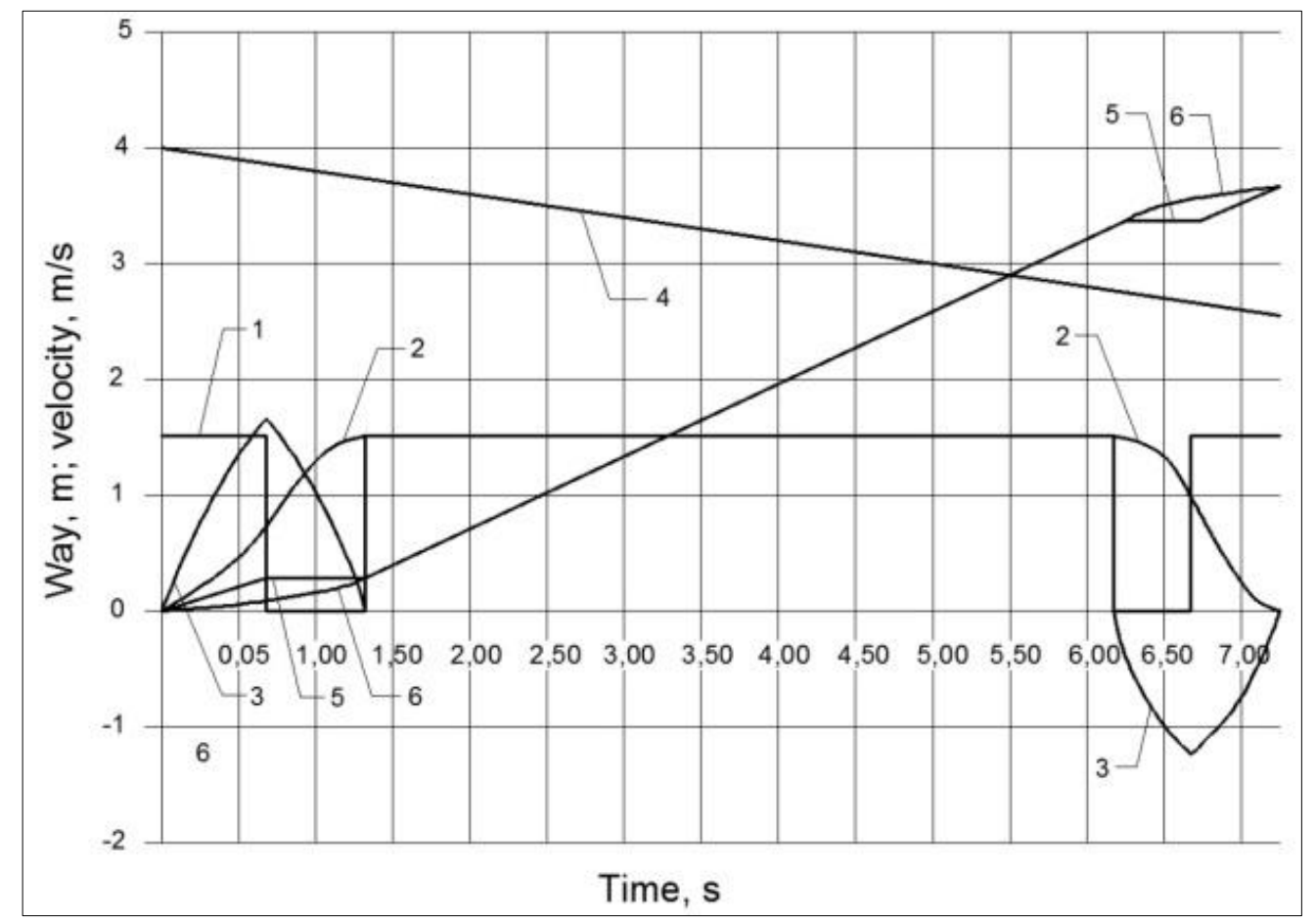

Fig. 5

As can be seen from the figure at such control the movement of the trolley comprises three stages. At the first stage that consists of two intervals (start and stop) the run of load to set horizontal velocity $\mathrm{V}_{\max }$ is fulfilled. The duration of the first stage is equal to $1,3 \mathrm{~s}$. The maximal deflection of load from vertical is $0,16 \mathrm{~m}$.

As the second stage the trolley and load move with equal velocity without the sway of load. The load continues its vertical movement.

At the third stage a maneuver is carried out, which also includes two intervals and provides full extinction of the sway to the moment of coming to the end point. The duration of stage is $1,0 \mathrm{~s}$. The maximal deflection of load is equal to $0,12 \mathrm{~m}$. The total duration of load transference is equal to $7,2 \mathrm{~s}$.

So, such quasi-optimal control at its separate stages has a simple structure, which permits more convenient realization. One more advantage of such control is low intensiveness of the sway and small connected with its dynamic loads on mechanisms (what promote increasing of its lasting).

But by the main index-quick-action the quasi-optimal control mutably gives in to strictly optimal (so the duration of cargo transference at the optimal control is equal to $6,66 \mathrm{~s}$ and at quasi-optimal $-7,2 \mathrm{~s}$ or for $8 \%$ more). 


\section{CONCLUSIONS}

Three methods of calculation of the time optimal control of light bridge cranes at transference of load on the flexible rope suspension are considered.

The first method is intended for calculation of load transference on the rope with the constant length. In that case, the simulation model of the crane can be presented by the system of linear differential equations, which permits to evaluate analytically general character of the optimal control and permits to define its structure (quantity and durations of intervals) by the comparatively simple numerical method.

The second method, which does not require linearity of the simulation model equations, is based on a very complex procedure of simultaneous integration of equations of auxiliary function $\varphi_{1}$ of Hamilton and non-linear equations of the simulation model. The results received by two methods coincide.

It gives possibility to affirm that the second method in spite of its complexity is reliable enough.

The third method is the modification of the second one, connected with taking into account combination of working movements of the crane. The method is intended for utilization at definition of the time-optimal control of the trolley at transference of load on the rope with the varying length.

The same methods and programs can be used for calculations of near to optimal by effective kinds of the control with only five intervals, when oscillations of load are damped at the transient stages (horizontal acceleration and deceleration) and must be a part of its way, the load on varying rope is moving synchronously with the trolley without a sway (fig.4). But at such kinds of control $\mathrm{V}_{5}(\mathrm{t})$ the total time of transference becomes noticeably greater (almost by $10 \%$ in dependence of distance) than at the optimal control. The simplicity of such control makes it convenient for utilization but the decline of productivity may be decisive at choice of the control for automatic realization.

\section{SUMMARY}

Three methods of calculation of the time optimal control of light bridge cranes are considered.

The first method is intended for calculation of load transference on the rope with the constant length. In that case, the simulation model of the crane can be presented by the system of linear differential equations. The second method, which does not require linearity of simulation model equations, is based on very complex procedure of simultaneous integration of equations of auxiliary function $\varphi 1$ of Hamilton and non-linear equations of the simulation model. The third method is intended for utilization at definition of the time-optimal control of the trolley at the transference of load on the rope with the varying length. The third method is modification of the second one. The same methods and programs can be used for calculations of near to optimal by effective kinds of the control with only five intervals. These are the methods of calculation of the time optimal control of light bridge cranes. 


\section{REFERENCES}

1. Bao Qifan. Research and development of automatic bulk cargo equipment in modern parts. Port Technology International. 2009. Vol. 41. pp. 88-93.

2. Chernousjko F. L. Upravlenie kolebaniyami / F. L. Chernousjko, L. D. Akulenko, B. N. Sokolov. M.: Nauka,1980. 384 pp.

3. Verschov T. Cranes-Design, Practise and Maintenance (Chapter 6. Sway and Swiny; Automation the trolley travelling mechanism). Professional Engineering Publishing. 2002. pp. 167-173.

4. Strel'tsov P. M. Gashenie kolebaniy gruza pri ego peremeshchenii na podvese peremennoy dliny. Visnyk ONMU. 2012. № 35. pp. 179-189.

5. Boltyanskiy V. G. Matematicheskie metody optimal'nogo upravleniya. M.: Nauka, 1969. 408 p.

6. Vasil'ev F. P. Chislennye metody resheniya ekstremal'nykh zadach. M.: Nauka, 1988. 545 p.

\section{Information about the authors:} Streltsov P. M.,

Candidate of Technical Sciences, Associated Professor at the Department of Hoisting and Transport Machines and Engineering of Port Technological Equipment, Odessa National Maritime University 34, Mechnikov str., Odessa, Ukraine ORCID ID: orcid.org/ 0000-0002-9260-4375

Lehetska I. P., Lecturer at the Department of Hoisting-and-Transport Machines and Port Technological Equipment Engineering, Odessa National Maritime University 34, Mechnikov str., Odessa, Ukraine ORCID ID: orcid.org/0000-0002-0858-0770

Labunska T. S.,

Lecturer at the Department of Hoisting-and-Transport Machines and Port Technological Equipment Engineering, Odessa National Maritime University 34, Mechnikov str., Odessa, Ukraine ORCID ID: orcid.org/0000-0003-4980-3771 